\title{
Terence J. Bazzett: An introduction to behavior genetics
}

\author{
MacMillan, 2008, HB, 476 pp, ISBN 978-0-87893-049-4
}

\author{
Arianna Di Florio $\cdot$ Nick Craddock
}

Published online: 21 June 2009

(C) Springer-Verlag 2009

This useful and nicely produced book is addressed mainly to students in the natural and social sciences and aims to provide a foundation for someone entering the field of behavior genetics. It will appeal to this audience because it uses a clear, accessible writing style and has an excellent standard of visual presentation with many explanatory figures. Furthermore, frequent boxes supplement the text to give entertaining and sometimes thought-provoking digressions or examples. In general, there are simple explanations with many practical examples that will help the reader to translate the theoretical concepts into practical issues. At the end of each chapter, the author provides a useful, concise summary followed by review questions and exercises addressed to the basic concepts.

The book is organized into four parts. The introductory part presents a simple but comprehensive overview on behavior genetics, outlining not only the biological and methodological basis, but also the roots and the history of this field, making a strong case for the importance of Human Genome Project. This carries over into many later chapters. The second part provides comprehensive summaries about concepts in the inheritance of simple and complex traits, considers some basic concepts of evolution and discusses the basic methods used to study hereditability, both in animal models and in human research. The third part outlines the genetic influences on behavior and behavioral and psychiatric disorders. The final part of the book focuses on physical health issues related to psychopathology and then considers genetic counseling, pharmacogenomics and gene therapy. The book ends with a useful glossary of terms.

As with all such introductory books, clarity and simplicity can represent both a strength and a potential weakness when dealing with a topic as complicated as behavior genetics. This is particularly true for the sections on psychopathology where the treatment is rather uneven and does not address many of the phenotypic complexities. However, after reading this book as a primer, the interested reader could go on to read other more detailed texts, taking forward their basic grounding in the area.

In summary, given the simple explanations and pleasing presentation, students in behavioral science can be expected to benefit from this book. It will help them gain a grounding in the key concepts and issues.

\footnotetext{
A. D. Florio $(\square) \cdot$ N. Craddock

Department of Psychological Medicine and Neurology, School of Medicine, Cardiff University, Cardiff CF14 4XN, UK e-mail: DiFlorioA@ cardiff.ac.uk

N. Craddock

e-mail: CRADDOCKN@ cardiff.ac.uk
} 